\title{
Antitumor activity of Pulsatilla koreana extract in anaplastic thyroid cancer via apoptosis and anti-angiogenesis
}

\author{
BYUNG HEE PARK * , KYUNG HEE JUNG ${ }^{*}$, MI KWON SON, JU HYEON SEO, \\ HEE-SEUNG LEE, JU-HEE LEE and SOON-SUN HONG \\ Department of Biomedical Sciences, College of Medicine, Inha University, \\ Sinheung-dong, Jung-gu, Incheon 400-712, Republic of Korea
}

Received June 8, 2012; Accepted October 31, 2012

DOI: $10.3892 / \mathrm{mmr} .2012 .1166$

\begin{abstract}
Plants or herb extracts have emerged as a novel approach to controling various diseases, including cancers. Among them, Pulsatilla koreana extract (PKE) has been widely used as an anti-inflammatory agent and for treating dysentery in traditional Korean and Chinese medicine. However, the effect of PKE as a cancer drug candidate has been less reported. Thus, we investigated the effect of PKE on cell growth and its mechanism in anaplastic thyroid cancer (ATC) cells. In this study, PKE suppressed the growth of ATC cells in a dose-dependent manner. Additionally, PKE induced apoptosis by increasing expression of cleaved PARP and caspase- 3 in ATC cells. The apoptotic effect of PKE was confirmed by diamidino-2-phenylindole (DAPI) and terminal deoxynucleotidyltransferase-mediated nick end labeling (TUNEL) assay, showing apoptotic body and DNA fragmentation. In addition, PKE decreased the expression of hypoxia-inducible factor $1 \alpha(\mathrm{HIF}-1 \alpha)$ and vascular endothelial growth factor (VEGF) as well as inhibiting tube formation and migration of human umbilical vein endothelial cells (HUVECs). Furthermore, in vivo studies showed that PKE significantly inhibited tumor growth and weight in a mouse xenograft model. Taken together, the present study demonstrated that PKE induced apoptosis, as well as inhibiting cell growth and angiogenesis in ATC cells. We suggest that PKE is a potent anticancer drug candidate for the treatment of thyroid cancer.
\end{abstract}

Correspondence to: Professor Soon-Sun Hong, Department of Biomedical Sciences, College of Medicine, Inha University, 3-ga, Sinheung-dong, Jung-gu, Incheon 400-712, Republic of Korea E-mail: hongs@inha.ac.kr

*Contributed equally

Key words: Pulsatilla koreana extract, apoptosis, angiogenesis, thyroid cancer, anaplastic thyroid cancer

\section{Introduction}

Thyroid cancer is a common endocrine malignant tumor, accounting for $1 \%$ of human cancers. These tumors have been classified as well-differentiated thyroid carcinomas, including the papillary (PTC) and follicular (FTC) types, which account for more than $95 \%$ of thyroid cancers, or anaplastic thyroid carcinomas (ATC), which account for just $1-5 \%$ of thyroid malignancies. Differentiated thyroid cancers such as PTC and FTC usually grow slowly and are highly curable using a combination of surgery, radioiodine ablation and thyroid-stimulating hormone (TSH)-suppressive therapy. However, ATC is a lethal disease with a median survival period of 6 months subsequent to diagnosis (1-4). ATC is a malignant undifferentiated neoplasm, without the thyroid differentiations. This malignant tumor is usually well-advanced by the time of diagnosis, with an average tumor size of $8 \mathrm{~cm}$. Ninety percent of patients with ATC have extraglandular spread at the time of diagnosis, while $75 \%$ of them develop distant metastases $(5,6)$. Consequently, ATC cases are staged as stage IV in the American Joint Commission on Cancer system (7). Whether primary chemotherapy/radiotherapy results in a longer survival period compared to the outcomes of primary surgical intervention remains controversial. Nevertheless, no effective therapeutic regimen has been identified for ATC as yet. This may be partly due to the rarity of this carcinoma; however, it also reflects the inadequacy of the available treatment options and suggests an urgent need for the development of novel treatment strategies (8).

Pulsatilla koreana is a perennial plant that grows around Korea and China and is used in traditional Chinese herbal medicine. It has been used to treat amoebic dysentery, malaria and internal hemorrhoids (9). Pulsatilla koreana extract (PKE) contains various bioactive compounds; some of these are capable of lowering the blood pressure, and also demonstrate anti-inflammatory effects and anti-acne activities against aerobic bacteria and fungi (10). In addition, several studies have reported that the compounds in PKE have anticancer effects in human melanoma, colon and lung cancers (11). PKE has also recently been reported to show anticancer effects in hepatocellular carcinoma (12). Although ATC is the most lethal disease among thyroid cancers, research on ATC treatment is insufficient. Therefore, this study aimed to investigate the anticancer activity of PKE in ATC, and the mechanism 
whereby PKE affects apoptosis and angiogenesis, as previously described, with regard to the pathogenesis of cancer.

\section{Materials and methods}

Extraction of PKE. The powdered roots of Pulsatilla koreana were extracted as described in our previous study (12). Briefly, they were extracted using 50\% ethanol, while the final extracts were concentrated in vacuo to yield a light brown residue. The residue was suspended in acetone, then centrifuged, and the resulting supernatant was removed to obtain a brown precipitate. The precipitate was poured into water and subsequently filtered to remove the insoluble portion. The filtrate was concentrated into a brown mass.

Cells and materials. Human ATC cell line $8505 \mathrm{c}$ was purchased from the Japanese Collection of Research Bioresources (JCRB, Shinjuku, Japan), while SNU-80 was purchased from the Korean Cell Line Bank (Seoul, Korea). 8505c cells were cultured in minimum essential medium Eagle (MEM) (Gibco-BRL, Carlsbad, CA, USA), whereas SNU-80 cells were cultured in RPMI-1640 (Gibco-BRL), supplemented with $10 \%$ fetal bovine serum (FBS, Gibco-BRL) and $1 \%$ penicillin/streptomycin. Cultures were maintained at $37^{\circ} \mathrm{C}$ in a $\mathrm{CO}_{2}$ incubator with a controlled humidified atmosphere composed of $95 \%$ air and 5\% $\mathrm{CO}_{2}$. Human umbilical vein endothelial cells (HUVECs) were grown in $0.2 \%$ gelatin-coated $75-\mathrm{cm}^{2}$ flasks in endothelial cell growth medium (ECGM) 2, containing its supplement mixture at $37^{\circ} \mathrm{C}$. 3-(4,5-dimethylthiazol-2-yl)-2,5-diphenyl tetrazolium bromide (MTT) and proteinase K were purchased from SigmaAldrich (St. Louis, MO, USA). RNase A was purchased from Qiagen (Valencia, CA, USA).

Cell viability assay. Cell viability was performed through an MTT assay. Briefly, 8505c and SNU-80 cells were plated at a density of $7 \times 10^{3}$ cells/well on 96 -well plates overnight. The media were removed, and cells were treated with either saline as a control or various concentrations of PKE followed by incubation for $48 \mathrm{~h}$. After that, MTT solutions were added to each well and incubated for $4 \mathrm{~h}$ at $37^{\circ} \mathrm{C}$. The formazan crystals that formed were dissolved in dimethyl sulfoxide (DMSO). Absorbance was measured using a microplate reader at $540 \mathrm{~nm}$. Three replicate wells were used for each analysis.

Western blot analysis. The cells were washed with ice-cold phosphate-buffered saline (PBS), then lysed with TNN buffer containing $1 \%$ Triton X-100, $1 \%$ Nonidet P-40, as well as the following protease and phosphatase inhibitors: aprotinin $(10 \mathrm{mg} / \mathrm{ml})$, leupeptin $(10 \mathrm{mg} / \mathrm{ml})$ (ICN Biomedicals, Inc., Asse-Relegem, Belgium), phenylmethylsulfonyl fluoride (1.72 mM), NaF (100 mM), $\mathrm{NaVO}_{3}(500 \mathrm{mM})$ and $\mathrm{Na}_{4} \mathrm{P}_{2} \mathrm{O}_{7}$ $(500 \mathrm{mg} / \mathrm{ml})$ (Sigma-Aldrich). Equal amounts of protein were separated by SDS-PAGE then transferred onto PVDF. Immunostaining of the blots was performed using the primary antibodies, followed by the secondary antibodies conjugated to horseradish peroxidase and detection by enhanced chemiluminescence reagent (ELPS, Seoul,Korea). The primary antibodies were monoclonal antibodies: anti-HIF-1 $\alpha$ (BD Biosciences, San Jose, CA, USA), anti-vascular endothelial growth factor (VEGF) (Santa Cruz Biotechnology, Inc., Santa Cruz, CA,
USA), anti-Bax, anti-Bcl-2 (Santa Cruz Biotechnology, Inc.), anti-cleaved caspase-3 and anti-cleaved poly ADP-ribose polymerase (PARP; Cell Signaling Technology, Inc., Danvers, MA, USA). The secondary antibodies were purchased from Amersham Biosciences, Inc., (Piscataway, NJ, USA) The bands were visualized with the ECL Plus system (Amersham Pharmacia Biotech, Inc., Piscataway, NJ, USA).

DAPI staining and terminal deoxynucleotidyltransferasemediated nick end labeling (TUNEL) assay. 8505c cells were plated onto $18-\mathrm{mm}$ cover glasses in MEM medium at $\sim 70 \%$ confluence for $24 \mathrm{~h}$. The cells were then treated with PKE at $100 \mu \mathrm{g} / \mathrm{ml}$ for $24 \mathrm{~h}$. They were fixed in $2 \%$ ice-cold paraformaldehyde (PFA), washed with PBS, then stained with $2 \mu \mathrm{g} / \mathrm{ml}$ of 4,6-diamidino-2-phenylindole (DAPI) for $20 \mathrm{~min}$ at $37^{\circ} \mathrm{C}$. The DAPI-stained cells were examined under a fluorescent microscope analyzing nuclear fragmentation. TUNEL was performed following the manufacturer's instructions for TUNEL kit (Chemicon, Temecula, CA, USA).

Tube formation assay. Matrigel (BD Biosciences, Franklin Lakes, NJ, USA) was polymerized for $30 \mathrm{~min}$ at $37^{\circ} \mathrm{C}$. HUVECs were suspended in ECGM2 medium, containing $50 \mathrm{ng} / \mathrm{ml}$ VEGF at a density of $3 \times 10^{4}$ cells $/ \mathrm{ml}$, and $0.2 \mathrm{ml}$ of cell suspension was added to each Matrigel-coated well, with or without the concentrations of PKE indicated for $10 \mathrm{~h}$. The morphological changes of the tube formation were observed under a phasecontrast microscope and photographed at magnification, x200.

Migration assay. HUVECs, plated on culture dishes of $60 \mathrm{~mm}$ diameter at $90 \%$ confluence, were wounded with a 2-mm razor blade and marked at the injury line. Subsequently, the cells that peeled off were removed with PBS and the wounded HUVECs were incubated in media with $50 \mathrm{ng} / \mathrm{ml}$ VEGF, $1 \mathrm{mM}$ thymidine (Sigma-Aldrich) and/ or PKE. HUVECs were allowed to migrate for $16 \mathrm{~h}$ and were then rinsed with PBS, followed by fixation with methanol.

Immunofluorescence. $8505 \mathrm{c}$ cells and HUVECs were seeded on 18 -mm glass plates in growth medium at $\sim 70 \%$ confluence for $24 \mathrm{~h}$. The cells were treated with $\mathrm{CoCl}_{2}$ for $1 \mathrm{~h}$. Subsequently, PKE was added to the medium and incubated for $6 \mathrm{~h}$. They were fixed in $2 \%$ ice-cold PFA, and washed with PBS. Immunostaining of the cells was performed using the primary antibodies, followed by the secondary antibodies conjugated to FITC (Vector Laboratories, Burlingame, CA, USA) or TRITC (Vector Laboratories) then stained with $2 \mu \mathrm{g} / \mathrm{ml}$ of DAPI for $20 \mathrm{~min}$ at $37^{\circ} \mathrm{C}$. Each slide was observed using a confocal laser scanning microscope (Olympus, Tokyo, Japan).

Tumor xenograft study. Male nude mice were obtained from the Central Animal Laboratory, Inc. (Seoul, Korea). Animal care and experimental procedures were in accordance with the approval and guidelines of the Inha Institutional Animal Care and Use Committee (INHA IACUC) of the Medical School of Inha University (Incheon, Korea). The animals were fed standard rat chow and tap water ad libitum, and were kept under $12 \mathrm{~h}$ dark/light cycle at $21^{\circ} \mathrm{C}$. Male nude mice $(6$ weeks; weight, 20-22 g) were randomized to three groups (control, PKE 125 and PKE $250 \mathrm{mg} / \mathrm{kg}$ ). 8505c cells were harvested 

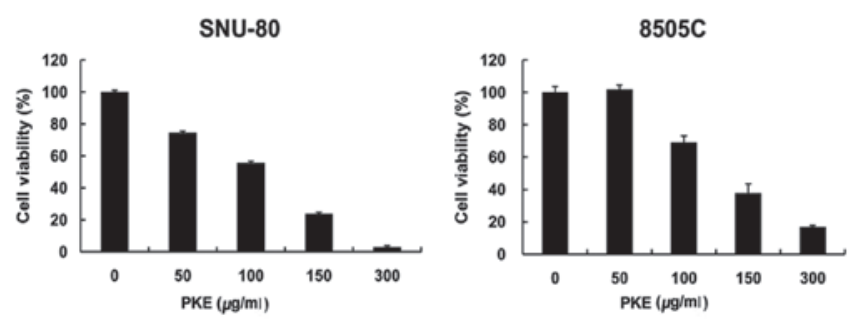

Figure 1. Effect of Pulsatilla koreana extract (PKE) on the proliferation of anaplastic thyroid cancer (ATC) cells is shown. Cytotoxic effects of PKE on ATC cells, SNU-80 and 8505 c ATC cells were measured by an MTT assay. ATC cells were seeded on 96-well culture plates. After incubation for $24 \mathrm{~h}$, the cells were treated with varying concentrations of PKE. After incubation for $48 \mathrm{~h}$, they were subjected to an MTT assay. Results are expressed as percent cell proliferation relative to the control. Data are represented as mean $\pm \mathrm{SD}$ from the triplicate wells.

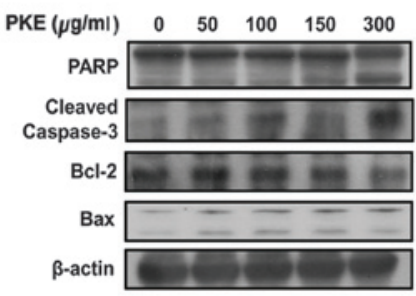

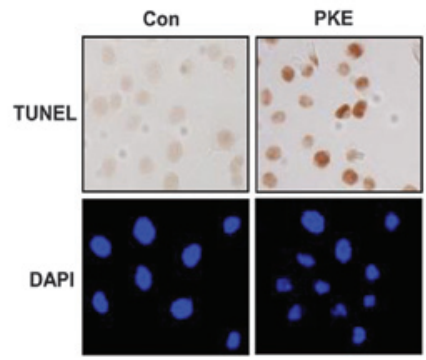

Figure 2. Effect of Pulsatilla koreana extract (PKE) on apoptosis of $8505 \mathrm{c}$ anaplastic thyroid cancer (ATC) cells. (A) Expression of PARP, cleaved caspase-3, Bax and Bcl-2 were determined by western blot analysis in cells treated with PKE at the indicated doses for $48 \mathrm{~h}$. (B) Induction of apoptosis by PKE was conducted by TUNEL and DAPI staining, which were photographed at magnification, $\mathrm{x} 200$.
A

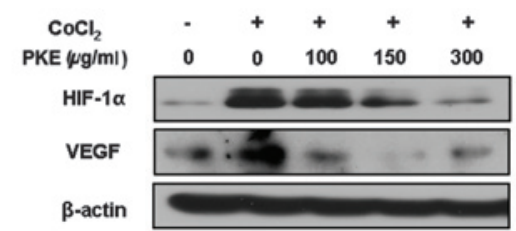

B

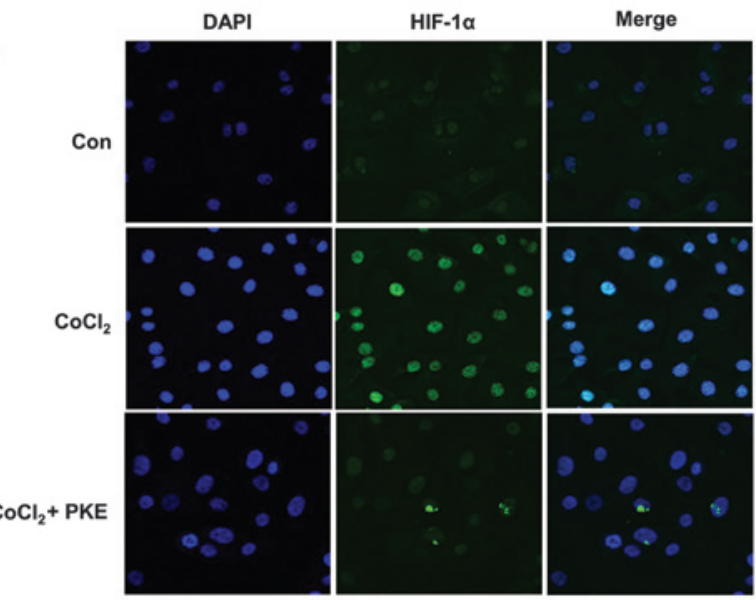

Figure 3. Effect of Pulsatilla koreana extract (PKE) on angiogenesis of $8505 \mathrm{c}$ cells. Expression of HIF-1 $\alpha$ and VEGF by PKE in hypoxia-induced $8505 \mathrm{c}$ anaplastic thyroid cancer (ATC) cells $\left(\mathrm{CoCl}_{2}, 100 \mu \mathrm{M}\right)$. (A) Western blot analysis and (B) immunofluorescence.
A

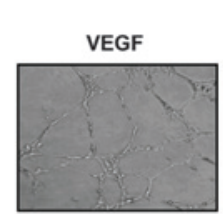

Con

B

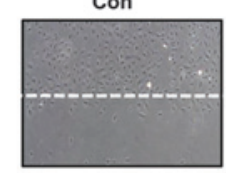

C<smiles>CC(C)(C)C1CCCCC1</smiles>

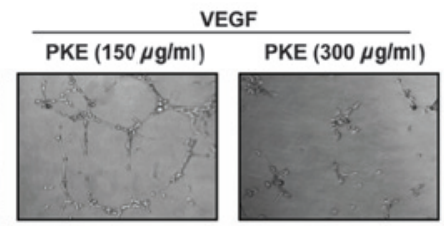

VEGF

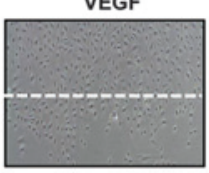

VEGF
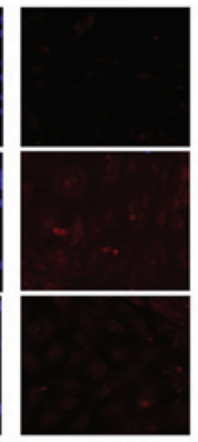

VEGF + PKE $(150 \mu \mathrm{g} / \mathrm{ml})$

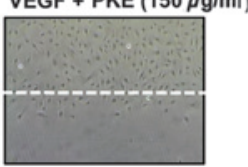

Merge

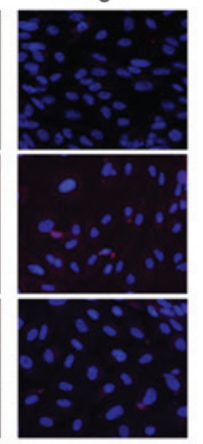

Figure 4. Effects of Pulsatilla koreana extract (PKE) on angiogenesis in human umbilical vein endothelial cells (HUVECs). (A) HUVECs were plated on Matrigel (200 $\mu 1 /$ well) and treated with various concentrations of PKE. Capillary tube formation was assessed after $10 \mathrm{~h}$. Tube formation was observed under a phase-contrast microscope and photographed at magnification, $\mathrm{x} 400$. (B) Effects of PKE on migration in vitro. HUVECs were plated at $90 \%$ confluence and scratched with a yellow tip. After wounding, cells were washed with PBS and incubated in ECGM2 containing 2\% FBS and $50 \mathrm{ng} / \mathrm{ml}$ recombinant human VEGF, $1 \mathrm{mM}$ thymidine and/or PKE $(100 \mu \mathrm{g} / \mathrm{ml})$. ECGM2 medium with $2 \%$ FBS was used as a negative control. Data represent the mean of at least three independent experiments performed in triplicate. (C) Immunocytochemistry of VEGF in hypoxia-induced HUVECs $\left(\mathrm{CoCl}_{2}, 100 \mu \mathrm{M}\right)$.

and mixed with PBS (200 $\mu 1 /$ mouse), and then inoculated into one flank of each nude mouse $\left(1 \times 10^{7}\right.$ of 8505 c cells $)$. When the tumors had reached a volume of $\sim 50 \mathrm{~mm}^{3}$, the mice were given a daily intraperitoneal injection of PKE (125 and $250 \mathrm{mg} / \mathrm{kg}$, treated group) or vehicle (200 $\mu 1 \mathrm{PBS}$, control group) for 28 days. The tumor dimensions were measured twice a week using a digital caliper, while the tumor volume was calculated using the formula: $\mathrm{V}=$ length $\mathrm{x}$ width $^{2} \mathrm{x} 0.5$. At the end of the experiment, the mice were sacrificed and the tumors were excised and weighed.

Statistical analysis. Data were expressed as the mean $\pm \mathrm{SD}$, and statistical analysis was performed using ANOVA and an unpaired Student's t-test. $\mathrm{P} \leq 0.05$ was considered to indicate a statistically significant difference. Statistical calculations were performed using SPSS software for Windows operating system (version 10.0; SPSS, Chicago, IL, USA).

\section{Results}

Inhibition of ATC cell growth by PKE. The effect of PKE on the viability of two ATC cell lines (8505c and SNU-80) was examined. ATC cells were incubated in media containing $50-300 \mu \mathrm{g} / \mathrm{ml}$ of PKE for $48 \mathrm{~h}$. The results showed that cell 
A

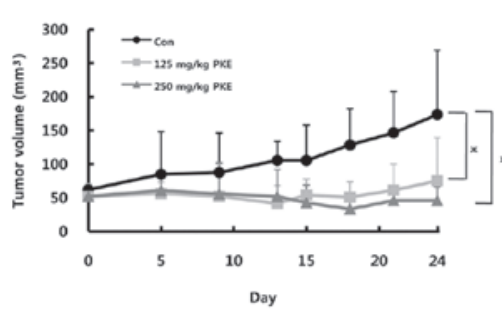

C

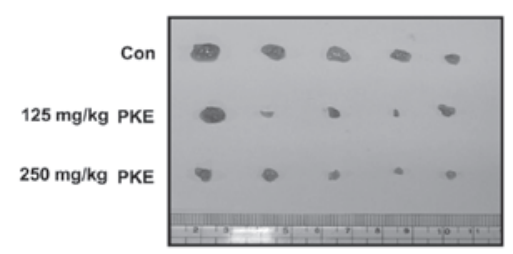

B

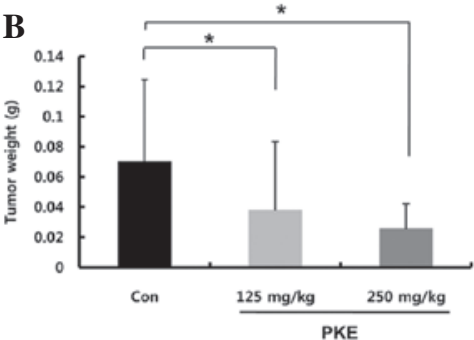

D

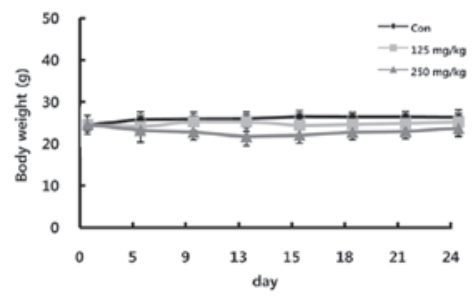

Figure 5. In vivo effect of Pulsatilla koreana extract (PKE) on 8505c anaplastic thyroid cancer (ATC) mouse xenograft model is shown. (A) Tumor growth of 8505c ATC xenograft in nude mice. The mice were injected subcutaneously with $8505 \mathrm{c}$ of ATC cells (1x107 cells/200 $\mu 1$ PBS) on the flank. (B) Weight of isolated tumors. (C) Photograph of tumors isolated from mouse xenograft. (D) Average body weight of nude mice. Data are represented by the mean \pm SD $(\mathrm{n}=5$ or 6$) .{ }^{*} \mathrm{P}<0.05$ vs. control.

growth was inhibited by PKE treatment in a dose-dependent manner (Fig. 1). The $\mathrm{IC}_{50}$ for growth inhibition was $120 \mu \mathrm{g} / \mathrm{ml}$ on SNU-80 and $140 \mu \mathrm{g} / \mathrm{ml}$ on $8505 \mathrm{c}$ ATC cells.

Effects of PKE on apoptotic cell death in 8505c ATC cells. To identify the apoptotic effect of PKE in 8505c ATC cells, the expression of Bax and Bcl-2, as well as the cleavage of PARP and caspase- 3 was measured by western blot analysis with PKE for $48 \mathrm{~h}$. PKE was found to lead to the upregulation of Bax and cleaved PARP and caspase-3 and a downregulation of Bcl-2 in 8505c ATC cells in a dose-dependent manner (Fig. 2A). These results showed that PKE induced cell apoptosis in 8505c ATC cells. When treated with PKE $(100 \mu \mathrm{g} / \mathrm{ml})$, the $8505 \mathrm{c}$ ATC cells presented the morphological features of apoptotic cells, such as DNA fragmentation and perinuclear apoptotic bodies by DAPI (Fig. 2B). The results of the TUNEL also exhibited PKE-induced apoptosis by causing DNA strand breaks.

Effects of PKE on angiogenesis. HIF-1 $\alpha$ and VEGF are important angiogenic factors in tumor progression. Thus, the effect of PKE on the hypoxia-induced HIF-1 $\alpha$ and VEGF expressions was examined. The cells were treated with varying concentrations of PKE under hypoxia-like conditions induced by $\mathrm{CoCl}_{2}(100 \mu \mathrm{M})$ for $18 \mathrm{~h}$. As shown in Fig. 3A, the HIF-1 $\alpha$ expression was increased under hypoxic conditions, whereas PKE treatment at 100-300 $\mu \mathrm{g} / \mathrm{ml}$ inhibited the hypoxia-induced HIF- $1 \alpha$ expression in a dose-dependent manner. Additionally, VEGF expression was increased under the hypoxia-like conditions, while PKE inhibited VEGF expression at a dose of $100-300 \mu \mathrm{g} / \mathrm{ml}$. Consistent with the results of the western blot analysis, immunofluorescence using a confocal microscope also showed that PKE inhibited HIF-1 $\alpha$ expression (Fig. 3B). In addition, the anti-angiogenic potential of PKE was examined using HUVECs. During an in vitro tube formation assay, PKE was observed to have inhibited the formation of vessel- like structures, comprising the elongation and alignment of the cells at the indicated concentrations (Fig. 4A). Cell migration is critical for endothelia cells to form blood vessels in angiogenesis and is necessary for tumor growth and metastasis. Thus, a wound migration assay was carried out to examine the effect of PKE on cell migration. Notably, when the endothelial cells were wounded and incubated in media with $50 \mathrm{ng} / \mathrm{ml}$ VEGF and $1 \mathrm{mM}$ thymidine in the presence of PKE $(100 \mu \mathrm{g} / \mathrm{ml})$ for $16 \mathrm{~h}$, subsequent to PKE treatment the wound was unable to heal (Fig. 4B). In addition, since VEGF is one of the critical factors of the tube formation and migration of HUVECs, we investigated whether PKE affects the expression of VEGF under hypoxic conditions in HUVECs using immunofluorescence. As expected, PKE decreased VEGF expression under hypoxia (Fig. 4C). These results showed that PKE prevented the tube formation and migration of endothelial cells while inhibiting the VEGF expression, suggesting that PKE had a potent anti-angiogenic property.

Inhibition of tumor growth by PKE in a mouse xenograft model. Based on these findings demonstrating a strong efficacy of PKE against 8505c ATC cells, the in vivo efficacy of PKE against the 8505c ATC cells was next examined in a nude mouse xenograft. As shown in Fig. 5A, PKE induced dose-dependent tumor growth inhibition at the doses of 125 or $250 \mathrm{mg} / \mathrm{kg}$ for 24 days, when compared to the control group. PKE administration at the doses of 125 and $250 \mathrm{mg} / \mathrm{kg}$ resulted in a significant reduction of the tumor volume (57 and $74 \%$, respectively) in nude mice. Consistent with this finding, the tumor weight isolated from PKE-treated groups was decreased by 54 and $36 \%$ at the PKE doses of 125 and $250 \mathrm{mg} / \mathrm{kg}$, respectively, compared to the control (Fig. 5B and C, P<0.05). No difference in the body weight of PKE-treatment groups was observed, compared to the control group (Fig. 5D), indicating that PKE had a low toxicity in mice at curative doses. These 
results demonstrated the in vivo antitumor efficacy of PKE against ATC without any apparent sign of toxicity.

\section{Discussion}

Thyroid cancer, particularly ATC, is an undifferentiated, fast-growing malignancy, for which novel therapeutic approaches are needed. Several studies are currently being conducted with a view to investigating the anticancer effects of plants or their components, given their lower toxic properties as natural products (13). In cancer therapy, approximately $70 \%$ of the effective drugs are products of natural origin or may be traced back to their pharmachophores (14). In this study, therefore, the anticancer efficacy of PKE and its mechanism in ATC were investigated. The results showed that PKE inhibited tumor growth and induced apoptosis both in vitro and in vivo. PKE also suppressed angiogenesis by decreasing the expression of HIF-1 $\alpha$ and VEGF.

Apoptosis, which is also known as programmed cell death, plays a critical role in treating cancer (15). Caspases are a conserved family of enzymes that bring cells to apoptosis. Of these, caspase- 3 is one of the key components of apoptosis, being responsible either partially or completely for the proteolytic cleavage of various key proteins, such as PARP, a protein repairing DNA and maintaining genomic DNA integrity $(16,17)$. Thus, the anticancer effects of PKE were first investigated through the mechanism of apoptosis in $8505 \mathrm{c}$ cells. In this study, we observed that PKE increased the expression of cleaved caspase-3 and PARP, leading to apoptotic cell death. These apoptotic effects of PKE were confirmed by the results of TUNEL and DAPI staining. The cells keep the homeostasis of the anti-apoptotic regulators, including $\mathrm{Bcl}-2$, and pro-apoptotic regulators, such as Bax, to maintain the proper survival and turnover. In this study, a dose-dependent increase of Bax and decrease of Bcl-2 were observed in PKE-treated 8505c cells. These results implied that PKE-induced apoptosis is likely to be an important factor in the suppression of tumor growth.

Angiogenesis is a prerequisite for tumor growth, as well as metastatic spread, and involves the recruitment of blood vessels by growing primary tumors or metastases. It is initiated by angiogenic factors, such as VEGF $(18,19)$. HIF-1 $\alpha$, an upstream signal molecule of VEGF, has been targeted as a major regulator of angiogenesis in various types of cancers (20). Since VEGF may be inhibited through the inactivation of HIF-1 $\alpha$, the HIF-1 $\alpha$ /VEGF pathway may be a candidate target of therapeutic strategy for ATC. These results showed that PKE inhibited HIF- $1 \alpha$ and VEGF expression under $\mathrm{CoCl}_{2}$-induced hypoxia conditions in $8505 \mathrm{c}$ cells. Additionally, extracts of natural plants, such as green tea or Ginkgo biloba have been reported to show anti-angiogenic effects through the inhibition of VEGF. Moreover, the in vitro anti-angiogenic effect of PKE was supported by the inhibition of HUVEC cell migration and tube formation, indicating that PKE inhibited angiogenesis through VEGF, as well as through targeting endothelial cells directly. Consequently, being a natural product, PKE has great potential as an anticancer agent.

In conclusion, although the anticancer activity of PKE and its mechanism have not been investigated in thyroid cancer, findings of the present study demonstrate the anticancer effects of PKE in ATC cells involved in the induction of apoptosis, as well as of anti-angiogenesis by inhibition of VEGF via HIF-1 $\alpha$ suppression. These findings suggest that PKE may be a potential candidate for cancer therapy against ATC.

\section{Acknowledgements}

This study was supported by the Inha University Grant and the Korean Health Technology R\&D Project (A120266), Ministry of Health \& Welfare, and the National Research Foundation of Korea (NRF) funded by the Ministry of Education, Science and Technology (NRF 2012-0002988, 2012R1A2A2A01045602).

\section{References}

1. Catalano MG, Poli R, Pugliese M, Fortunati N and Boccuzzi G: Emerging molecular therapies of advanced thyroid cancer. Mol Aspects Med 31: 215-226, 2010.

2. Fassnacht M, Kreissl MC, Weismann D and Allolio B: New targets and therapeutic approaches for endocrine malignancies. Pharmacol Ther 123: 117-141, 2009.

3. Patel KN and Shaha AR: Poorly differentiated and anaplastic thyroid cancer. Cancer Control 13: 119-128, 2006.

4. Sakorafas GH, Sampanis D and Safioleas M: Cervical lymph node dissection in papillary thyroid cancer: current trends, persisting controversies, and unclarified uncertainties. Surg Oncol 19: e57-e70, 2010.

5. Shimaoka K, Schoenfeld DA, DeWys WD, Creech RH and DeConti R: A randomized trial of doxorubicin versus doxorubicin plus cisplatin in patients with advanced thyroid carcinoma. Cancer 56: 2155-2160, 1985.

6. Ahuja S and Ernst H: Chemotherapy of thyroid carcinoma. J Endocrinol Invest 10: 303-310, 1987.

7. Fleming ID, Phillips JL, Menck HR, Murphy GP and Winchester DP: The National Cancer Data Base report on recent hospital cancer program progress toward complete American Joint Committee on Cancer/TNM staging. Cancer 80: 2305-2310, 1997.

8. Herbst RS: ZD1839: targeting the epidermal grow th factor receptor in cancer therapy. Expert Opin Investig Drugs 11: 837-849, 2002.

9. Bae K: The medicinal plants of Korea. Kyo-Hak Press, Seoul, 1999.

10. Lee HS, Beon MS and Kim MK: Selective growth inhibitor toward human intestinal bacteria derived from Pulsatilla cernua root. J Agric Food Chem 49: 4656-4661, 2001.

11. Bang SC, Lee JH, Song GY, Kim DH, Yoon MY and Ahn BZ: Antitumor activity of Pulsatilla koreana saponins and their structure-activity relationship. Chem Pharm Bull (Tokyo) 53: 1451-1454, 2005.

12. Hong SW, Jung KH, Lee HS, et al: Apoptotic and anti-angiogenic effects of Pulsatilla koreana extract on hepatocellular carcinoma. Int J Oncol 40: 452-460, 2012.

13. Yin F, Giuliano AE and Van Herle AJ: Growth inhibitory effects of flavonoids in human thyroid cancer cell lines. Thyroid 9: 369-376, 1999.

14. Cragg GM, Newman DJ and Yang SS: Natural product extracts of plant and marine origin having antileukemia potential. The NCI experience. J Nat Prod 69: 488-498, 2006.

15. O'Connor L, Huang DC, O'Reilly LA and Strasser A: Apoptosis and cell division. Curr Opin Cell Biol 12: 257-263, 2000.

16. Riedl SJ and Shi Y: Molecular mechanisms of caspase regulation during apoptosis. Nat Rev Mol Cell Biol 5: 897-907, 2004.

17. Krishnakumar R and Kraus WL: The PARP side of the nucleus: molecular actions, physiological outcomes, and clinical targets. Mol Cell 39: 8-24, 2010.

18. Folkman J: What is the evidence that tumors are angiogenesis dependent? J Natl Cancer Inst 82: 4-6, 1990.

19. Hanahan D and Folkman J: Patterns and emerging mechanisms of the angiogenic switch during tumorigenesis. Cell 86: 353-364, 1996.

20. Bolat F, Haberal N, Tunali N, Aslan E, Bal N and Tuncer I: Expression of vascular endothelial growth factor (VEGF), hypoxia inducible factor 1 alpha (HIF-lalpha), and transforming growth factors beta1 (TGFbeta1) and beta3 (TGFbeta3) in gestational trophoblastic disease. Pathol Res Pract 206: 19-23, 2010 . 